\title{
Effect of wing deformation on the aerodynamic performance of flapping wings: fluid- structure interaction approach
}

\begin{abstract}
Wing stiffness is very crucial in augmenting aerodynamic forces in flapping wing flyers. In this work, the effect of wing deformation was studied using three-dimensional numerical analysis (two-way fluid structure interaction), coupling the flow solver (FLUENT) and the structural $(A B A Q U S)$ solver via the $M p C C I$ platform. Three different degrees of bending stiffness corresponding to rigid, flexible, and highly flexible case wings were investigated. Moreover, the wings were tested for both low Reynolds number $(\mathrm{R}=9,000)$ and high Reynolds number $(\mathrm{R}=40,000)$, at a flapping frequency of $9 \mathrm{~Hz}$ corresponding to an angle of attack (AoA) ranging from $\alpha=0$ to $50^{\circ}$. The results of mean aerodynamic lift and drag coefficients showed good agreement between numerical and experimental findings. Also, the time-averaged lift-to-drag ratio reveals that the highly flexible wing exhibited the best overall aerodynamic performance when compared to the rigid and flexible wing.
\end{abstract}

Keyword: Wing deformation; Aerodynamic performance; Flapping wings; Fluid-structure interaction approach 